\title{
Neueste Entwicklungen im vietnamesischen Recht der Auslandsinvestitionen
}

\author{
Von Arno Wohlgemuth
}

Am 30. Juni 1990 verabschiedete die Nationalversammlung der SR Vietnam ein Änderungsgesetz zum Gesetz über ausländische Investitionen in Vietnam (InvG) vom 29. Dezember 19871, durch das Artikel 2 und 26 InvG neugefaßt wurden.2 Bisher war es vietnamesischen Privatpersonen und vietnamesischen privaten Wirtschaftseinheiten 3 nicht möglich, direkt ein Gemeinschaftsunternehmen oder eine Arbeitsgemeinschaft mit einem ausländischen Investor in Vietnam zu gründen. Sie konnten sich an einer solchen Verbindung nur in der Weise beteiligen, daß sie einer staatlichen oder halbstaatlichen vietnamesischen Wirtschaftsorganisation Kapital zur Verfügung stellten, dessen sich diese Organisation bediente, um ihrerseits allein ein Joint Venture mit einem ausländischen Partner einzugehen. 4 Dieses Hindemis für Privatpersonen und -gesellschaften ist nunmehr beseitigt. Während zudem unter dem InvG 1988 nur ein Zwei-Partner-Joint-Venture5 gestattet war, ist heute ein "ménage à trois" und mehr, also ein Multi-Partner-Joint-Venture,

1 Siehe dazu: Arno Wohlgemuth, Das vietnamesische Investitionsgesetz von 1988, WGO-Monatshefte für Osteuropäisches Recht (= WGO-MfOR) 30 (1988), 241-254. Zum früheren vietnamesischen Recht der Auslandsinvestitionen vgl. Brun-Otto Bryde, Der Kodex über Auslandsinvestitionen der Sozialistischen Republik Vietnam, VRÜ 11 (1978), S. 103 ff.

2 Summary of Worldbroadcasts, Part 3, Far East, BBC Monitoring, Reading/Berkshire, U.K., v. 3.7. 1990 Nr. 0806/B S. 4-5 (= SWB FE/0806/b/4-5 v. 3.7.1990).

3 Vgl. auch Direktive des Ministerrates Nr. 82-CT vom 19.3.1990 über nichtstaatliche Genossenschaften, SWB FE/0727/B/4 v. 31.3.1990; zur ersten privaten Handelsgesellschaft in Ho-ChiMinh-Stadt, SWB FE/0221/B/4 v. 4.8.1988; Arno Wohlgemuth, Das vietnamesische Gesetz über Privatgewerbe von 1990, WGO-MfOR 33 (1991).

4 Vgl. auch Canice Chew-Ming Chan, The Developing Legal Framework for Foreign Investment in Vietnam, ICSID Review - Foreign Investment Law Joumal 4 (1989), 276-296 (280); Luu Van Dat, La loi sur les investissements étrangers au service du développement de coopération, Revue internationale de droit contemporain 2/1988, 81-88; Marie-Françoise Labouz, A propos de la nouvelle loi vietnamienne sur les investissements étrangers, Joumal du droit intemational 117 (1990), 93 104; W. Gary Vause, Doing Business with Vietnam - Prospects and Concerns for the 1990s, Florida International Law Joumal 4 (Spring 1989), 231-287; Neill T. Macpherson, Foreign Investment in The Philippines, Thailand and Vietnam, International Business Lawyer 17 (1989), 233234; John Gillespie, Foreign Investment in SR Vietnam Revisited, International Business Lawyer 18 (1990), 416-424.

5 Zu Joint Ventures allgemein siehe Klaus Langelfeld-Wirth (Hrsg.), Joint Ventures im intemationalen Wirtschaftsverkehr - Praktiken und Vertragstechnik intemationaler Gemeinschaftsuntemehmen, Heidelberg 1990. 
erlaubt. ${ }^{6}$ Der andere Teil der Änderungen bezieht sich auf den Rahmen der Körperschaftsteuer für die ausländische Seite eines Investitionsunternehmens. 7

Neben dieser Novelle zum InvG hat das Dekret des Ministerrates Nr. 139-HDBT vom 5. September 1988 über die Einzelheiten der Durchführung des Gesetzes über ausländische Investitionen in Vietnam (InvD) umfassende Detailregelungen und Ergänzungen zum InvG kodifiziert.8 Das 113 Artikel zählende Dekret regelt in 11 Kapiteln ausführlich die allgemeinen Grundlagen (Kap. I), die vertragliche Unternehmens-Kooperation (Kap. II), das Joint Venture (Kap. III), die Investitionsunternehmen in ausschließlich ausländischer Hand (Kap. IV), die Betriebstätigkeit in Vietnam (Kap. V), das für Investitionsunternehmen beachtliche Arbeitsrecht (Kap. VI), steuer- (Kap. VII), devisen-9 (Kap. VIII) und bilanzrechtliche10 Fragen (Kap. IX) und schließlich Angelegenheiten des Imports und Exports 11 , der Zölle12 sowie der Einreise und Freizügigkeit von ausländischen Investoren und deren ausländischen Mitarbeitern innerhalb Vietnams (Kap. X).

Als Staatsbehörde für die Verwaltung ausländischer Investitionen (Kap. V InvG) ist nach Art. 6 Abs. 1 InvD das Staatskomitee für Kooperationen und Investitionen (SKKI) eingerichtet, daneben ist dem Ministerium für Außenwirtschaftsbeziehungen (MfAW) nach Art. 6 Abs. 2 InvD die Rolle des Adressaten für Investitionsprojekte zugewiesen. An mehreren Stellen ist im InvD die Rede von Gesellschaften für Investitionsdienste (Art. 7 Abs. 2, 69 Buchst. b) InvG). Für diese Consulting-Firmen ist die Direktive des Minister-

6 Minister Comments of Amended Foreign Investment Law, SWB FE/0820/B/1-2 v. 19.7.1990.

7 SWB FE/0795/B/4 v. 20.6.1990; SWB FE/0796/B/2 v. 21.6.1990; Vietnam Gives Bigger Role to Private Investment, Far Eastem Economic Review (= FEER) v. 26.7.1990.

8 Von einem Kontrollrecht der staatlichen Sicherheitsbehörden gegenüber ausländischen Investitionsunternehmen, wie es durch das sowjetische Dekret vom 26.1.1991 für die UdSSR statuiert wurde (vgl. KGB to Check Joint Ventures, Intemational Herald Tribune (=IHT) v. 4.2.1991, S. 1), ist in Vietnam nichts bekannt. Weitere investitionsrechtliche Bestimmungen Vietnams sind auf englisch abgedruckt in: Bundesstelle für Außenhandelsinformation - Dokument Nr. R 108/90 v. 10.12.1990.

9 Vgl. Verordnung des Ministerrates vom Oktober 1988 über Devisenkontrolle, SWB FE/W0053/ A/15-16 v. 23.11.1988; Direktive des Vorsitzenden des Ministerrates vom 18.8.1989 über Devisentransaktionen, SWB FE/W0104/C1/1 v. 22.11.1989; New State Bank Policy on Foreign Currency, SWB FE/0914/B6 v. 6.11.1990.

10 Im Hinblick auf die in der Buchführung zu verwendenden Maßeinheiten ist das 39 Artikel umfassende Dekret des Staatsrates vom 16.7.1990 über Maßeinheiten zu beachten, SWB FE/0825/B/2 v. 25.7.1990.

11 Vgl. Beschluß des Ministerrates Nr. 223-HDBT vom 28.12.1989 betreffend die Steuertabelle für den Im- und Exporthandel, SWB FE/W0112/A/8 v. 24.1.1990; vgl. auch Arno Wohlgemuth, Das Recht des Außenhandelskauf vertrages in Vietnam, IPRax 1990, 192-195.

12 Hier ist die Verordnung des Staatsratex vom 24.2.1990 über die Rollregelung zu beachten, SWB FE/0713/B/8-9 v. 15.3.1990; auch Entscheidung des Vorsitzenden des Ministerrates Nr. 133-CT vom 13.5.1989 über Zölle und Besteuenung des Grenzverkehrs, SWB FE/0465/B/4 v. 24.5.1989. 
rates Nr. 280-CT vom November 1990 maßgeblich. ${ }^{13}$ Gegenwärtig versucht Vietnam zusammen mit der australischen Gesellschaft Nelson Wheeler Indochina in Hongkong ein Consulting-Büro für Investitionsprojekte in Vietnam zu gründen. 14

Ein Joint Venture ist als GmbH (Art. 33 InvD) mit einem Verwaltungsrat und einem Generaldirektor (Art. 34-39 InvD) zu errichten. Dasselbe gilt für ein ausländisches Investitionsunternehmen ohne vietnamesische Beteiligung (Art. 55 InvD). Die GmbH muß eine solche des vietnamesischen Rechts sein. Bestimmungen über die $\mathrm{GmbH}$ (Société à responsabilité limitée) fanden sich früher nur in den Kodifikationen aus der französischen Kolonialära.15 Heute gilt das Gesetz über Handelsgesellschaften vom 21. Dezember 1990.16 Über Einzelheiten des Gesetzes ist hier noch nichts bekannt.

Im Joint-Venture-Vertrag haben die Parteien eine Bestimmung sowohl über die zuständige Schieds- oder Gerichtsbehörde (in Frage kommen kann etwa auch das Handelsarbitrageinstitut bei der Handelskammer von Stockholm oder das Regionale Arbitragezentrum des Asiatisch-Afrikanischen Justizkonsultativausschusses in Kuala Lumpur/Malaysia)17 für eventuelle Streitigkeiten 18 aus der Partnerschaft als auch über das in dem Streitfall anzuwendende Recht zu treffen (Art. 24 Buchst. (k), 53 InvD). Bezüglich der Rechtswahl ist ihnen anders als nach rotchinesischem Recht19 keine Beschränkung auferlegt. Ob die kollisionsrechtliche Verweisungsfreiheit nur für den Basisvertrag gilt oder darüber hinaus auch das Gesellschaftsrecht des in der Form einer GmbH tätigen Joint-Venture-Unternehmens erfaßt, muß nicht unbedingt im ersteren Sinne zu beantworten sein. Schließlich stand den einheimischen (vietnamesischen) Parteien in Vietnam unter französischer Dominanz zumindest für den Kolonialkonflikt zwischen indigenem Recht und dem der Metropole (conflit colonial) eine Option (professio iuris) zugunsten französischen Gesellschaftsrechts - wie es auch in anderen französischen Besitzungen (z.B. Algerien) der Fall war - zu.20

13 Directive Issued on Foreign Investment Services, SWB FE/0918/B/6 v. 10.11.1990.

14 Robin Agarwal, Hanoi Reaches Out, FEER v. 19.7.1990, S. 58.

15 Vgl. Le Tai Trien, Republic of Viet-Nam (Stand: Oktober 1971), in: Intemational Encyclopedia of Comparative Law, Vol. I (National Reports), hrsg. v. Viktor Knapp, Tübingen, The Hague, Paris, S. R-21.

16 National Assembly Announces New Laws, SWB FE/0962/B/2 v. 5.1.1991; SWB FE/0806/B/5 v. 3.7.1990; SWB FE/0812/B/3 v. 10.7.1990; SWB FE/0797/B/2 v. 22.6.1990; SWB FE/0834/B/3 v. 4.8.1990.

17 Vgl. dazu auch Chan a.a.O. (Fn. 4), $285 \mathrm{f}$.

18 Die Ordnung des Staatsrates vom 10.1.1990 über Wirtschaftsarbitrage spielt hier keine Rolle, SWB FE/0662/B/1 v. 15.1.1990.

19 Vgl. dazu Arno Wohlgemuth, Von Juche zu Joint Venture: Das Recht der nordkoreanisch-ausländischen Gemeinschaftsuntemehmen, WGO-MfOR 30 (1988), 282.

20 Vgl. André Dureteste, Cours de droit de l'Indochine, Paris 1938, S. 182. 
Bezüglich der steuerrechtlichen Regelungen ${ }^{21}$ sind die neuerlassenen Gesetze über Umsatzsteuer, über Einkommensteuer und über Sonderverbrauchssteuer jeweils vom 30. Juni 1990 zu berücksichtigen. 22

Die Bestimmungen über Arbeitsverhältnisse in Investitionsunternehmen (Kap. VI)23 sind um das Dekret des Staatsrates über Arbeitsverträge vom 30. August 1990 ergänzt worden.24 Bedeutsam in diesem Zusammenhang ist auch das Gesetz über Gewerkschaften vom 30. Juni 1990.25

Im InvD finden sich Ausführungs- oder Ergänzungsregeln weder zu den Investitionsschutznoch Eigentumsgarantien zusätzlich zu denjenigen im InvG (Kap. III). Ebensowenig sind eigentumsrechtliche Fragen berührt. Hier ist nur Kapitel IV des Bodengesetzes vom 29. Dezember 198726 beachtlich, das Regelungen über die Nutzung von Grund und Boden durch ausländische Organisationen und Einzelpersonen und Joint Ventures sowie vietnamesisch-ausländische Arbeitsgemeinschaften enthält.27 Für Investitionen im Bereich der Exploration und Ausbeutung mineralischer Rohstoffe finden sich in dem acht Kapitel und 36 Artikel umfassenden Gesetz des Staatsrates über Bodenschätze vom 28. Juli 198928 Sonderbestimmungen. Für Fischereikooperationen gilt das Protokoll des Ministerrates über die Regelung des Fischfangs durch Ausländer und ausländische Schiffe in vietnamesischen Gewässem vom 22. Dezember 1990.29

Vom 11. bis 15. März 1991 fand das 1. Investitionsforum30 für Vietnam in Ho-Chi-MinhStadt unter der Schirmherrschaft von UNIDO (UN Industrial Development Organization),

21 In der VR China etwa ist ein einheitliches Körperschaftsteuergesetz für ausländische Investitionsunternehmen (auch Joint Ventures) am 9.4.1991 erlassen worden, vgl. Amendments Proposed to Tax Law for Foreign Concems, SWB FE/1008/C1/1-2 v. 28.2.1991; Han Guojian, Attuning Taxation to Investment Climate, Beijing Review, March 11-17, 1991, 22-25; Yang Xiaobin, New Tax Law Favours Foreign Enterprises, Beijing Review, May 6-12, 1991, $26-27$.

22 Siehe dazu näheres bei Arno Wohlgemuth, Das vietnamesische Gesetz über Privatgewerbe von 1990, a.a.O. (Fn. 3); Vietnam - Income Tax, Bulletin of Legal Developments 1991 No. 1, S. 9.

23 Vgl. auch Phan Hien, Les droits de l'homme dans l'entreprise au Vietnam, Revue juridique et politique, indépendence et coopération 43 (1989), 874-879.

24 Regulation on Inheritance and Labour Contracts, SWB FE/0873/B/2 v. 19.9.1990; Phap lenh hop dong lao donc, Nhan Dan v. 17.9.1990, S. 2.

25 SWB FE/0806/B/4-5 v. 3.7.1990; SWB FE/0558/B/2 v. 11.9.1989.

26 Law on Land, SWB FE/00600/C2/1-8 v. 28.1.1988.

27 Der Beschluß des Ministerrates über die Entschädigung bei Nutzungsänderung von Land- und forstwirtschaftlichen Grundstücken vom Juni 1990 bezieht sich nicht auf ausländische Investitionsunternehmen, SWB FE/0791/B/3 v. 15.6.1990.

28 Law on Mineral Resources Summarised, SWB FE/W0092/A/12-13 v. 30.8.1989.

29 Council of Ministers Issues New Fishing Regulations, SWB FE/0963/B/6 v. 7.1.1991.

30 Zum Bedarf Vietnams an Auslandsinvestitionen für die Teewirtschaft siehe: Vietnam will zum "Teekönigreich" aufsteigen, Frankfurter Allgemeine Zeitung v. 5.3.1991, S. 12. 
UNDP (UN Development Programme) und der SKKI vor Delegationen aus 39 Staaten statt. Dort wurde vermeldet, daß seit Erlaß des InvG im Jahre 1987 über 200 Investitionsprojekte mit einem Gesamtkapital von 1,4 Billionen US-Dollar eröffnet worden seien.31 $21 \%$ dieser Investitionen gehen auf das Konto Frankreichs.32 An zweiter Stelle steht Kanada, gefolgt von Großbritannien und Belgien33; Japan34 nimmt den fünften Platz ein.35 Ende 1989 waren $80 \%$ aller Vorhaben wegen der besseren Infrastruktur im Süden des Landes angesiedelt. 36 Allein in Ho-Chi-Minh-Stadt waren Lizenzen bis Juli 1990 für insgesamt 33 ausländische Investitionsanträge erteilt worden mit einem registrierten Kapital von 230,5 Mio US-Dollar. Die Hälfte der Investoren sind aus Asien, der Rest kommt aus Europa37, Nordamerika und Australien. Wenn alle Unternehmen im ehemaligen Saigon ihren Betrieb aufnehmen, werden sie 20.000 Arbeitsplätze schaffen und mit einer Jahresdurchschnittsproduktion von 330 Mio US-Dollar aufwarten können. 38 Investoren beklagen

31 Vietnam Tries to Lure Foreign Investment, IHT v. 12.3.1991, S. 17; Investment Forum, SWB FE/ 1020/B/5 v. 14.3.1991; Nguyen Van Linh, Outlines Vietnam's Policies to Investment Forum, WB FE/1027/B/6 v. 22.3.1991; zum Stand im Februar 1990 siehe SWB FE/W0115/A/9 v. 14.2.1990 (100 Projekte in Höhe von insgesamt 750 Mio US-Dollar).

32 France - Investment, SWB FE/W0163/A/11 v. 23.1.1991.

33 Am 24.1.1991 unterzeichneten Vietnam und Belgien ein Investitionsschutzabkommen in Hanoi, SWB FE/W0165/A/7 v. 6.2.1991.

34 Zum Rang Singapurs siehe SWB FE/W0161/A/16 v. 9.1.1991.

35 Foreign Investment: Japanese Report, SWB FE/W0161/A/15 v. 9.1.1991; Japanese Poised for Rush into Vietnam, IHT v. 3.1.1990, S. 9.

36 SWB FE/0586/B/6 v. 13.10.1989.

37 Siehe nunmehr: EC Considers Resuming Aid to Vietnam, IHT v. 12.2.1991, S. 11.

38 SWB FE/W0137/A/12 v. 18.7.1990; zu weiteren Investitionsuntemehmen siehe SWB FE/W0117/ A/9 v. 28.2.1990; SWB FE/W0065/i v. 22.2.1989 (Vertrag über Ölbohrungen mit BP); SWB FE/ W0054/A/8-9 v. 30.11.1988; SWB FE/W0051/A/15 v. 9.11.1988; SWB FE/W0049/A/11 v. 26.10.1988; SWB FE/W0048/A/19 v. 19.10.1099; IHT v. 21.3.1991, S. 17 (Speiseölraffinerie mit Malaysien); SWB FE/W0171/A/12 v. 20.3.1991 (Natriumglutamatfabrik mit Taiwan und optisches Untemehmen mit der UdSSR); SWB FE/W0170/A/9 v. 13.3.1991 (Ölfirma mit Thailand); SWB FE/W0168/A/8 v. 27.2.1991 (Kaffeeproduktion mit Deutschland; Gerberei mit Ungam und Fischverarbeitung mit der UdSSR); SWB FE/W0163/A/11 v. 23.1.1991 (Ölvertrag mit der australischen Broken Hill Proprietary Group Ltd. und mit der UdSSR; Niederlassung von Mitsubishi in Hanoi); SWB FE/W0167/A/7-8 v. 20.2.1991, SWB FE/W0160/A/11 v. 2.1.1991, SWB FE/0928/A3/6 v. 22.11.1990 (Schmierölfabrik mit der britischen Castro Ltd.; Joint-Venture-Bank mit der Bank Summa Indonesia); SWB FE/W0164/A/11 v. 30.1.1991 (Fleischverarbeitungsgesellschaft mit Taiwan); SWB FE/W0161/A/16 v. 9.1.1991 (Kautschukfabrik mit der UdSSR); SWB FE/W0160/ A/12 v. 2.1.1991 (Produktion von Gegenständen der Innendekoration mit Hongkong); SWB FE/ W0159/A/12-13 v. 19.12.1990 (Frankreich, Nordkorea); IHT v. 15./16.12.1990, S. 13 (Yasuda. Feuer- und Seeversicherungsgesellschaft/Japan); SWB FE/W0157/A/14 v. 5.12.1990 (Eßstäbchen mit Südkorea); weiter zu Japan, Taiwan und den Philippinen SWB FE/W0156/A/14 v. 28.11.1990, SWB FE/W0154/A/8-9 v. 14.11.1990; zu Kanada (Reisproduktion) SWB FE/W0152/A/13 v. 31.10.1990; siehe auch Article Views Foreign Investment in Vietnam, SWB FE/W0148/A/12 v. 3.10.1990; Vietnam Still Hoping For U.S. Oil Investment, IHT v. 21.9.1990, S. 19; Vietnam Says Foreign Investment Has Doubled, IHT v. 13.9.1990, S. 15; Oskar Weggel, Gesamtbericht - 
die Ergänzung der Gesetze der Zentralregierung durch die Regelungen der örtlichen Volksräte, loben aber die vietnamesischen Arbeitskräfte, deren Löhne zu den niedrigsten der Region zählen, für ihren Fleiß und ihre Disziplin.39

Auch in Vietnam macht die bürokratische Zentralplan- und Kontrollwirtschaft den Initiativen des privaten Sektors, der Wettbewerbsökonomie und dem objektiven Wirtschaftsrecht Platz.40 Das InvD hat zweifellos empfindliche Lücken des InvG geschlossen. Gesetzgeberischer Perfektionsdrang birgt freilich die Gefahr, durch Uberregulierungen im Investitionsrecht das Gegenteil dessen zu bewirken, was intendiert ist. Investitionsrecht als Kriseninterventionsgesetzgebung notleidender Nationalökonomien $41 \mathrm{muß}$ die unternehmerische Entscheidungsfreiheit und die Anlegerbereitschaft kompetenter und potenter Kapitalgeber mobilisieren und nicht durch administrative Regulative mehr als nach den Geboten einer "sozialistischen Marktwirtschaft" notwendig einschränken. Welches die probateste Investitionscharta ist, wird für jedes Land und seine erklärte Wirtschafts- und Sozialpolitik auf den jeweiligen Entwicklungsstufen unterschiedlich zu beantworten sein. Mit dem InvD von 1988 und den Novellierungen des InvG von 1990 hat der vietnamesische Gesetzgeber eine weitere Antwort gegeben, gewiß nicht die letzte. Jedenfalls sind die notwendigen Anpassungen des InvD an die Änderungen des InvG aus dem Jahre 1990 bereits angekündigt. 42

Vietnam, Kambodscha, Laos, Südostasien aktuell 1991/1, S. 48; The Far East and Australasia 1991, 22. Aufl. London 1990, S. 1104.

39 Investment - Stiff-Hinged Open Door, Asiaweek v. 8.2.1991, S. 56, 58; dort auch zur deutschen Germantex in Ho-Chi-Minh-Stadt. Im Februar 1991 hat Vietnam zum erstenmal an der "Internationalen Grünen Woche" in Berlin teilgenommen, SWB FE/0992/A1/1 v. 9.2.1991.

40 Gareth Porter, The Politics of "Renovation" in Vietnam, Problems of Communism May-June 1990, 72-88 (88); Douglas Pike, Vietnam in 1990, Asian Survey 31 (1991), 79-86.

41 Siehe zu Auslandshilfe und Investitionen, Vietnam, in: The Far East and Australasia 1991, a.a.O. (Fn. 38), S. $1103 \mathrm{ff}$.

42 Minister Comments on Amended Foreign Investment Law, SWB FE/0820/B/2 v. 19.7.1990. 
organs to comply fully with the Provisions of the Universal Declaration of Human Rights in all its policies and business towards securing the maintanance and upholding of the dignity of Man. This article comments on one of the High Court judgements which effectively applied established intemational standards prohibiting discrimination of women or discrimination made on the basis of sex.

\section{Recent Developments in the Vietnamese Law of Foreign Investment}

\section{By Arno Wohlgemuth}

According to a statement by Ngoc Xuan, president of the State Commission for Cooperation and Foreign Investment, from June this year Vietnam has licensed almost 300 foreign investment projects with capital of over $\$ 2$ billion in the past three years under the Foreign-investment Code 1987. This Code has been amended in June 1990. More than 50 detailed regulations have been promulgated under the code covering key areas like banking, finance, technology transfer, labour, wages and travel to and from Vietnam.

The Decree of the Council of Ministers No. 139/HDBT of September 5, 1988, regulating the implementation of the Law on Foreign Investment in Vietnam contains provisions of joint ventures, enterprises with $100 \%$ foreign capital and on business organisations. Other chapters deal with labour relations in enterprises with foreign invested capital, financial matters, foreign exchange control, accounts and audit, customs, immigration, residence and communications.

Further legislation concerning foreign direct investment in Vietnam, e.g. maritime law, aviation law and the oil and gas law, is envisaged. To boost protection of foreign investment in Vietnam the government is ready to sign agreements on investment protection and promotion and pacts on avoidance of double taxation (IHT of 26.6.1991, p. 17). 